\title{
Narrowing the Filter-Cavity Bandwidth in Gravitational-Wave Detectors via Optomechanical Interaction
}

\author{
Yiqiu Ma, ${ }^{1, *}$ Shtefan L. Danilishin, ${ }^{1}$ Chunnong Zhao, ${ }^{1, \dagger}$ Haixing Miao, ${ }^{2, \ddagger}$ W. Zach Korth, ${ }^{3}$ \\ Yanbei Chen, ${ }^{2}$ Robert L. Ward, ${ }^{4}$ and D. G. Blair ${ }^{1}$ \\ ${ }^{1}$ School of Physics, University of Western Australia, Western Australia 6009, Australia \\ ${ }^{2}$ Theoretical Astrophysics 350-17, California Institute of Technology, Pasadena, California 91125, USA \\ ${ }^{3}$ LIGO Laboratory, California Institute of Technology, Pasadena, California 91125, USA \\ ${ }^{4}$ Centre for Gravitational Physics, Department of Quantum Science, Australian National University, \\ Canberra ACT 0200, Australia
}

(Received 2 September 2013; revised manuscript received 6 March 2014; published 10 October 2014)

\begin{abstract}
We propose using optomechanical interaction to narrow the bandwidth of filter cavities for achieving frequency-dependent squeezing in advanced gravitational-wave detectors, inspired by the idea of optomechanically induced transparency. This can allow us to achieve a cavity bandwidth on the order of $100 \mathrm{~Hz}$ using small-scale cavities. Additionally, in contrast to a passive Fabry-Pérot cavity, the resulting cavity bandwidth can be dynamically tuned, which is useful for adaptively optimizing the detector sensitivity when switching amongst different operational modes. The experimental challenge for its implementation is a stringent requirement for very low thermal noise of the mechanical oscillator, which would need a superb mechanical quality factor and a very low temperature. We consider one possible setup to relieve this requirement by using optical dilution to enhance the mechanical quality factor.
\end{abstract}

DOI: 10.1103/PhysRevLett.113.151102

PACS numbers: $04.80 . \mathrm{Nn}, 42.50 . \mathrm{Wk}$

Introduction.-Advanced interferometric gravitational wave detectors, e.g., the advanced LIGO [1], advanced VIRGO [2], and KAGRA [3], are expected to be limited by quantum noise over almost the entire detection band. Further enhancement of the detector sensitivity requires manipulation of the optical field and the readout scheme at the quantum level. One approach proposed by Kimble et al. [4] is injecting frequency-dependent squeezed light into the main interferometer. A series of optical cavities is used to filter the squeezed light and to create proper rotation of the squeezing angle at different frequencies. In order to achieve a broadband reduction of quantum noise, the frequency scale of these filter cavities needs to match that of quantum noise of the main interferometer. For the advanced LIGO detector, the quantum noise is dominated by quantum radiation pressure noise at low frequencies and shot noise at high frequencies - the transition happens around $100 \mathrm{~Hz}$, which determines the required filter-cavity bandwidth.

The original proposal in Ref. [4] is using filter cavities of kilometer length. Recently, Evans et al. [5] proposed a more compact $(10 \mathrm{~m})$ filter cavity with $10^{5}$ finesse to achieve the required bandwidth. With such a high finesse, even small optical losses can degrade the squeezing. Therefore, the optical loss becomes the key limiting factor in the filer-cavity performance. Isogai et al. have experimentally demonstrated that the optical losses from current mirror technology are sufficiently small to build such a filter cavity that will be useful for the advanced LIGO detector [6]. However, if we want to further increase the compactness of the filter cavity, then the requirement for the optical loss becomes more stringent. In this case, one solution is to go beyond the paradigm of passive cavities. One proposed approach is to actively narrow the cavity bandwidth by using the electromagnetically induced transparency effect in a pumped atomic system [7]. In principle, the cavity can be made to be on the centimeter scale while still having a bandwidth comparable to a much longer highfinesse cavity. Additionally, with an active element, the cavity optical properties can be dynamically tuned by changing the power of the control pumping field. This has the advantage of allowing optimization of the filter cavity for different operational modes of the detector, where the quantum noise has different frequency dependencies, e.g., tuned versus detuned resonant sideband extraction in the case of the advanced LIGO detector.

The active atomic system is generally lossy, which will degrade the squeezing level. Here we propose to narrow the filter-cavity bandwidth using the optomechanical analogue of electromagnetically induced transparency, optomechanically induced transparency, which has recently been experimentally demonstrated by Weis et al. [8] and Teufel et al. [9]. In comparison with these optomechanically induced transparency experiments, we consider a different parameter regime and use an overcoupled cavity to attain the desired performance. The scheme integrated with the main interferometer is illustrated in Fig. 1. The filter cavity consists of a mirror-endowed mechanical oscillator with an eigenfrequency $\omega_{m}$ that is much larger than the cavity bandwidth $\gamma$. A control pump laser drives the filter cavity at frequency $\omega_{p}$, detuned from the cavity resonant frequency 
$\omega_{0}$ (also the laser frequency of the main interferometer) by $\omega_{m}-\delta$ with $\delta$ comparable to the gravitational-wave signal frequency $\Omega$. As we will show, the optomechanical interaction modifies the cavity response and gives rise to the following input-output relation for the sideband at $\omega_{0}+\Omega$ :

$$
\hat{a}_{\mathrm{out}}(\Omega) \approx \frac{\Omega-\delta-i \gamma_{\mathrm{opt}}}{\Omega-\delta+i \gamma_{\mathrm{opt}}} \hat{a}_{\mathrm{in}}(\Omega)+\hat{n}_{\mathrm{th}}(\Omega),
$$

where $\gamma_{\text {opt }}$ is defined as

$$
\gamma_{\mathrm{opt}}=\frac{4 P_{c} \omega_{0}}{m \omega_{m} c^{2} T_{f}}
$$

with $P_{c}$ being the intracavity power of the control field, $m$ the mass of the mechanical oscillator, and $T_{f}$ the transmissivity of the front mirror (the end mirror is totally reflective). The first term in Eq. (1) gives the input-output relation of a standard optical cavity with the original cavity bandwidth $\gamma$ replaced by $\gamma_{\text {opt }}$, which can be significantly smaller than $\gamma$ as well as dynamically tuned by changing the control beam power.

The second term $\hat{n}_{\text {th }}$ arises from the thermal fluctuation of the mechanical oscillator. It is uncorrelated with the input optical field $\hat{a}_{\text {in }}$ and therefore decoheres the squeezed light. In order for its effect to be small, we require

$$
\frac{8 k_{B} T}{Q_{m}}<\hbar \gamma_{\mathrm{opt}}
$$

with $Q_{m}$ the mechanical quality factor and $T$ the environmental temperature. Given the fact that the desired effective cavity bandwidth is $\gamma_{\mathrm{opt}} / 2 \pi \approx 100 \mathrm{~Hz}$, we have

$$
\frac{T}{Q_{m}}<6.0 \times 10^{-10} \mathrm{~K}
$$

This is challenging to achieve even with low-loss materials at cryogenic temperature. A possible solution is to use optical dilution, first proposed by Corbitt et al. [10-13]. It allows for a significant boost of the mechanical quality factor by using the optical field, to provide most of the restoring force. Later we illustrate its applicability for our purpose.

Optomechanical dynamics.-Here we provide the details behind Eq. (1) by analyzing the dynamics of the optomechanical filter cavity, starting from the standard linearized Hamiltonian [14,15]:

$$
\begin{aligned}
\hat{\mathcal{H}}= & \hbar \omega_{0} \hat{a}^{\dagger} \hat{a}+\frac{\hat{p}^{2}}{2 m}+\frac{1}{2} m \omega_{m}^{2} \hat{x}^{2}+\hbar \bar{G}_{0} \hat{x}\left(\hat{a}^{\dagger}+\hat{a}\right) \\
& +i \hbar \sqrt{2 \gamma}\left(\hat{a}^{\dagger} \hat{a}_{\text {in }} e^{-i \omega_{p} t}-\hat{a} \hat{a}_{\text {in }}^{\dagger} e^{i \omega_{p} t}\right) .
\end{aligned}
$$

In the Hamiltonian, $\hat{a}$ is the annihilation operator of the cavity mode and $\hat{a}_{\text {in }}$ is the annihilation operator for the input optical field (the squeezed light in our case), $\hat{x}(\hat{p})$ is the oscillator position (momentum), and $\bar{G}_{0}=\left[2 P_{c} \omega_{0} /\right.$ $(\hbar c L)]^{1 / 2}$ with $L$ being the cavity length. In the rotating frame at frequency $\omega_{p}$ of the control laser, the Heisenberg equation of motion reads

$$
\begin{gathered}
m\left(\ddot{\hat{x}}+\gamma_{m} \dot{\hat{x}}+\omega_{m}^{2} \hat{x}\right)=-\hbar \bar{G}_{0}\left(\hat{a}+\hat{a}^{\dagger}\right)+\hat{F}_{\mathrm{th}}, \\
\dot{\hat{a}}+(\gamma+i \Delta) \hat{a}=-i \bar{G}_{0} \hat{x}+\sqrt{2 \gamma} \hat{a}_{\mathrm{in}},
\end{gathered}
$$

where $\Delta \equiv \omega_{0}-\omega_{p}$ is the detuning frequency and we have included the mechanical damping and associated Langevin force $\hat{F}_{\text {th }}$. Solving these equations of motion in the frequency domain yields

$$
\begin{aligned}
& \hat{x}(\omega)=\chi_{m}(\omega)\left\{\hbar \bar{G}_{0}\left[\hat{a}(\omega)+\hat{a}^{\dagger}(-\omega)\right]+\hat{F}_{\text {th }}(\omega)\right\}, \\
& \hat{a}(\omega)=\chi_{c}(\omega)\left[-i \bar{G}_{0} \hat{x}(\omega)+\sqrt{2 \gamma} \hat{a}_{\text {in }}(\omega)\right] .
\end{aligned}
$$

We have defined the susceptibilities $\chi_{m} \equiv-\left[m\left(\omega^{2}-\omega_{m}^{2}+\right.\right.$ $\left.\left.i \gamma_{m} \omega\right)\right]^{-1}$ and $\chi_{c} \equiv[\gamma-i(\omega-\Delta)]^{-1}$.

Relevant parameter regime.-We consider the parameter regime leading to Eq. (1). This requires $\Delta=\omega_{m}-\delta$ with $\omega_{m} \gg \delta$, and the so-called resolved-sideband regime $\omega_{m} \gg \gamma$. Correspondingly, the lower sideband of the cavity mode $\hat{a}(-\omega)$ in Eq. (8) is negligibly small and can be ignored (we will analyze the effect of this approximation later). We therefore obtain [cf. Eqs. (8) and (9)]:

$$
\hat{a}(\omega) \approx \frac{\sqrt{2 \gamma} \hat{a}_{\mathrm{in}}(\omega)-i \bar{G}_{0} \chi_{m}(\omega) \hat{F}_{\mathrm{th}}(\omega)}{\chi_{c}^{-1}(\omega)+i \hbar \bar{G}_{0}^{2} \chi_{m}(\omega)} .
$$

Since we are interested in the signal sidebands around $\omega_{0}$, we rewrite the above expression in terms of $\Omega$ by using the equality $\omega=\Delta+\Omega$ [cf. the inset of Fig. 1]. Given $\Omega \approx \delta \ll \omega_{m}$, we have $\chi_{m} \approx-\left[2 m \omega_{m}\left(\Omega-\delta+i \gamma_{m}\right)\right]^{-1}$ and $\chi_{c} \approx \gamma^{-1}$. Together with $\hat{a}_{\text {out }}=-\hat{a}_{\text {in }}+\sqrt{2 \gamma} \hat{a}$, we obtain

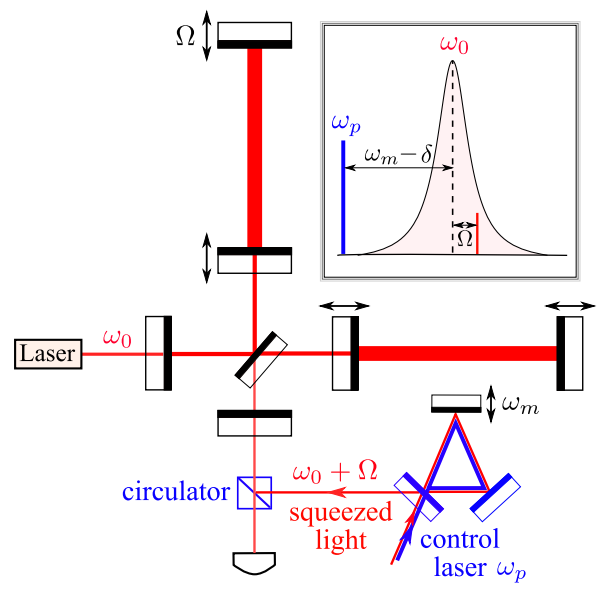

FIG. 1 (color online). Schematic showing the configuration for achieving frequency-dependent squeezing. 


$$
\hat{a}_{\text {out }}(\Omega) \approx \frac{\Omega-\delta+i \gamma_{m}-i \gamma_{\text {opt }}}{\Omega-\delta+i \gamma_{m}+i \gamma_{\text {opt }}} \hat{a}_{\text {in }}(\Omega)+\hat{n}_{\text {th }}(\Omega)
$$

with the additional noise term $\hat{n}_{\text {th }}$ defined as

$$
\hat{n}_{\mathrm{th}}(\Omega)=\frac{i \sqrt{2 \gamma} \gamma_{\mathrm{opt}} \hat{F}_{\mathrm{th}}(\Omega)}{\hbar \bar{G}_{0}\left(\Omega-\delta+i \gamma_{m}-i \gamma_{\mathrm{opt}}\right)} .
$$

When thermal damping rate $\gamma_{m}$ of the oscillator satisfies $\gamma_{m}<\gamma_{\text {opt }}$, we can ignore $\gamma_{m}$ and recover the input-output relation shown in Eq. (1).

To maintain coherence of the squeezed light, the fluctuations due to the thermal noise term $\hat{n}_{\text {th }}$ need to be much smaller than those due to the input field; equivalently, the quantum radiation pressure noise on the mechanical oscillator from the squeezed light needs to dominate over the thermal noise of the oscillator. Given the fact that $\left\langle\hat{F}_{\mathrm{th}}^{\dagger}(\Omega) \hat{F}_{\mathrm{th}}\left(\Omega^{\prime}\right)\right\rangle=4 m \gamma_{m} k_{B} T \delta\left(\Omega-\Omega^{\prime}\right)$, the requirement on the noise spectrum for $\hat{n}_{\text {th }}$ reads

$$
S_{\mathrm{th}}(\Omega)=\left(\frac{8 k_{B} T}{\hbar \gamma_{\mathrm{opt}} Q_{m}}\right) \frac{\gamma_{\mathrm{opt}}^{2}}{(\Omega-\delta)^{2}+\gamma_{\mathrm{opt}}^{2}}<1
$$

The thermal noise effect is maximal around $\Omega \sim \delta$, from which we obtain the condition shown in Eq. (3).

Effects of the optical loss and the finite cavity bandwidth.-Apart from the above-mentioned thermal noise, there are other decoherence effects: (i) the additional radiation pressure noise introduced by the optical loss, and also (ii) the effect of the lower sideband due to the finite cavity bandwidth, ignored in the resolved-sideband limit. Their effects are similar to the above thermal force noise; therefore, we can quantify their magnitude using the noise spectrum referred to the output. For the optical loss,

$$
S_{\epsilon}(\Omega)=\left(\frac{c \epsilon}{\gamma L}\right) \frac{\gamma_{\mathrm{opt}}^{2}}{(\Omega-\delta)^{2}+\gamma_{\mathrm{opt}}^{2}},
$$

where $\epsilon$ is the magnitude of the optical loss (e.g., $\epsilon=10^{-5}$ for $10 \mathrm{ppm}$ loss). Similarly, for the contribution from the lower sideband, we have

$$
S_{-\omega_{m}}(\Omega)=\left(\frac{\gamma}{\omega_{m}}\right)^{2} \frac{\gamma_{\mathrm{opt}}^{2}}{(\Omega-\delta)^{2}+\gamma_{\mathrm{opt}}^{2}} .
$$

These two need to be taken into account when estimating the performance of this optomechanical filter cavity.

Possible experimental scheme.-We have shown in Eq. (4) that the most significant issue is the thermal noise, which puts a stringent requirement on the mechanical system and the environmental temperature. As we mentioned earlier, one possible way to mitigate this is using optical dilution explored by Corbitt et al., in which the optical restoring force is due to the linear dependence of the radiation pressure force on the oscillator position. This scheme has a limitation from quantum backaction noise associated with a linear position response. Korth et al. [16] have shown how measurement-based feedback can cancel the quantum back action. Such a cancellation is, however, limited by the quantum efficiency of the photodiode for the measurement.

Here we consider optical dilution using a coupled cavity scheme, shown in Fig. 2, with a mirror-endowed oscillator placed in the middle of a Fabry-Pérot cavity, first implemented by Thompson et al. [17,18]. Interestingly, this scheme allows for an internal cancellation of the quantum backaction associated with a linear optical spring, and thus it avoids the limitation of the scheme in Ref. [16]. A detailed analysis is given in the Supplemental Material [19]. An intuitive picture behind this backaction evasion effect can be described as follows. The optical field on the left-hand side of the middle oscillator consists of two parts: (i) the immediate reflection from the oscillator and (ii) the transmitted field from the right-hand side, both containing the position information of the oscillator. The coupled cavity has a doublet resonance. It turns out that, when the trapping field is resonantly tuned to one of the two resonance frequencies and the end mirror is perfectly reflective, the position information from these two parts destructively interferes, resulting in a cancelation of the backaction.

A strong trapping beam can induce an optical spring frequency $\omega_{\mathrm{opt}} \gg \omega_{m 0}$ with

$$
\omega_{\mathrm{opt}}^{2}=\frac{2 P_{\mathrm{trap}} \omega_{0}^{\prime}}{m c^{2} \sqrt{T_{s}} T_{f}}
$$

where $P_{\text {trap }}$ and $\omega_{0}^{\prime}$ are the input power and optical frequency of the trapping beam, and $T_{s}$ and $T_{f}$ are the transmissivity of the mirror-endowed oscillator and the front mirror, respectively. The modified quality factor can be greatly boosted since the mechanical dissipation rate $\gamma_{m}$ is unchanged.

This optical dilution scheme also has its own limitations. First, in reality there is no perfectly reflective mirror and there is always some optical loss, so the above-mentioned cancellation cannot be perfect. The residual radiation pressure noise, referred to the output, is given by

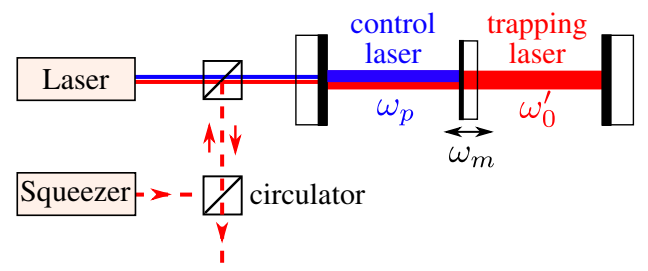

FIG. 2 (color online). Schematics of the coupled cavity setup for the filter cavity. 
TABLE I. Example parameter values.

\begin{tabular}{lcc}
\hline \hline Parameter & Description & Value \\
\hline$L$ & Filter-cavity length & $50 \mathrm{~cm}$ \\
$T_{f}$ & Front mirror transmissivity & $250 \mathrm{ppm}$ \\
$T_{s}$ & Transmissivity of oscillator & $3000 \mathrm{ppm}^{\mathrm{a}}$ \\
$P_{\text {trap }}$ & Trapping beam input power & $1.6 \mathrm{~mW}$ \\
$\lambda_{0}^{\prime}$ & Trapping beam wavelength & $532 \mathrm{~nm}$ \\
$m$ & Oscillator mass & $500 \mathrm{ng}$ \\
$\omega_{m_{0}} /(2 \pi)$ & Bare mechanical frequency & $200 \mathrm{~Hz}$ \\
$Q_{m_{0}}$ & Bare mechanical quality factor & $10^{8 \mathrm{~b}}$ \\
$T$ & Environmental temperature & $1 \mathrm{~K}$ \\
$P_{c}$ & Control beam intracavity power & $0.1 \mathrm{~mW}$ \\
$\lambda_{0}$ & Control beam wavelength & $1064 \mathrm{~nm}$ \\
$\gamma_{\text {opt }} /(2 \pi)$ & Effective cavity bandwidth & $100 \mathrm{~Hz}$ \\
\hline \hline
\end{tabular}

${ }^{\mathrm{a}}$ This value is only for the trapping beam; for the control field, the value is close to unity (limited by the optical loss), requiring a dichroic coating.

${ }^{\mathrm{b}}$ According to [23], the mechanical damping of some material structures are as small as $10^{-6} \mathrm{~Hz}$, which sets this possible value.

$$
S_{\epsilon}^{\mathrm{opt}}(\Omega)=\frac{4 \omega_{0}^{\prime} P_{\mathrm{trap}} \epsilon}{m \gamma_{\mathrm{opt}} \omega_{m} c^{2} T_{s} T_{f}} \frac{\gamma_{\mathrm{opt}}^{2}}{(\Omega-\delta)^{2}+\gamma_{\mathrm{opt}}^{2}} .
$$

Second, the optical spring effect is frequency dependent: $K_{\text {opt }}(\omega) \approx m \omega_{\text {opt }}^{2}-i m \Gamma \omega-m_{\text {opt }} \omega^{2}$. This tells us that the optical spring can modify not only the resonance frequency, but also the mechanical damping and the effective inertia (mass), which could induce instability. Lastly, finite absorption of the laser power in the oscillator will increase its temperature and may increase the thermal noise. The size of this effect, however, depends on the mechanical structure and the detailed loss mechanism.

An example.-We illustrate the requirements for experimentally realizing the optomechanical filter cavity using the optical dilution shown in Fig. 2 with some example parameters in Table I. These values are chosen after considering the above mentioned effects, which can cause decoherence to the squeezed light, such that

$$
S_{\mathrm{tot}}^{\max }=S_{\mathrm{th}}(\delta)+S_{\epsilon}(\delta)+S_{-\omega_{m}}(\delta)+S_{\epsilon}^{\mathrm{opt}}(\delta)<1 .
$$

In addition, once we fix the oscillator mass $m$ and transmissivity $T_{s}$, we can minimize $S_{\text {tot }}^{\max }$ by looking into the scaling of different parameters, which determines the trapping beam power $P_{\text {trap }}$, the front mirror transmissivity $T_{f}$, and the environmental temperature $T$. We end up with the following scaling of $S_{\mathrm{tot}}^{\max }$ in terms of the optical loss and the cavity length:

$$
S_{\text {tot }}^{\max } \approx 3 \times 10^{3} \epsilon^{4 / 5} / L^{2 / 5} .
$$

The resulting degradation to the squeezing factor due to the optical loss is shown in Fig. 3 for a cavity length of $50 \mathrm{~cm}$.

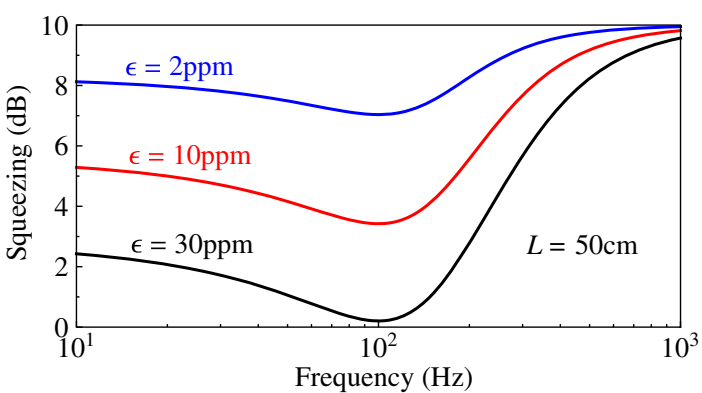

FIG. 3 (color online). Resultant squeezing level from injecting $10 \mathrm{~dB}$ of input squeezing into an optomechanical filter cavity using the optical-dilution scheme in Fig. 2, with parameters in Table I, for several values of the optical loss $\epsilon$.

In comparison to a passive filter cavity for which the performance degrades as $\epsilon / L[6]$, the optomechanical filter cavity using the optical-dilution scheme has a milder dependence on $L$, which yields the possibility of being small scale.

The mechanical dynamics are modified by the optomechanical interaction, and the new effective parameters of the oscillator are summarised in Table II. The optical spring shifts the mechanical resonant frequency from its bare value of $100 \mathrm{~Hz}$ to $20 \mathrm{kHz}$, which results in a 200-fold increase in the quality factor. Comparing Tables I and II, we can see that the negative optical damping and inertia do not pose an important problem.

We would like to point out that this scheme might not function as expected due to heating from the finite absorption of the laser power. The intracavity power of the trapping beam, given the listed parameter values, is around $10 \mathrm{~W}$. For $10 \mathrm{ppm}$ absorption, this amounts to $0.1 \mathrm{~mW}$ of heat deposited into the nanomechanical oscillator. We make an order-of-magnitude estimate in the Supplemental Material [19] and find this can create a nonuniform temperature distribution with a maximum around $10 \mathrm{~K}$ near the beam spot. Further detailed study is required to estimate how this nonuniform temperature distribution on the oscillator affects the total thermal noise. Specifically in this case, the dissipation mainly comes from the clamping point where the temperature is still low. If this nonuniform temperature distribution indeed introduces significant thermal noise, then alternative materials with higher thermal conductivity at low temperature would need to be manufactured.

TABLE II. Effective oscillator parameters.

\begin{tabular}{lcc}
\hline \hline$\omega_{\text {opt }} /(2 \pi)$ & Optical spring frequency [Eq. (16)] & $20 \mathrm{kHz}$ \\
\hline$Q_{m}$ & Final mechanical quality factor & $2 \times 10^{10}$ \\
$\Gamma /(2 \pi)$ & Optical (anti)damping rate [Eq. (A.9)] & $-8 \mathrm{mHz}$ \\
$m_{\mathrm{opt}}$ & Negative optical inertia [Eq. (A.10)] & $-8.5 \mathrm{pg}$ \\
\hline \hline
\end{tabular}


Conclusion.-We have considered the use of optomechanical interaction to narrow the bandwidth of a filter cavity for frequency-dependent squeezing in future advanced gravitational-wave detectors. However, due to the susceptibility to thermal decoherence, its feasibility is conditional on advancements in low-loss mechanics and optics.

We thank Huan Yang, David McCelland, Farid Khalili, Li Ju, and Jiayi Qin for fruitful discussions. Y. M., S. L. D., C.Z., R. L. W., and D. G. B. have been supported by the Western Australia Centers of Excellence program, and by the Australian Research Council; W. Z. K. is supported by NSF Grant No. PHY-0757058; H. M. and Y. C. are supported by NSF Grant No. PHY-1068881 and NSF CAREER Grant No. PHY-0956189.

*myqphy@gmail.com

†chunnong.zhao@uwa.edu.au

"haixing@caltech.edu

[1] B. P. Abbott et al., Rep. Prog. Phys. 72, 076901 (2009).

[2] Advacnced VIRGO project, https://wwwcascina.virgo.infn .it/advirgo/.

[3] K. Somiya, Classical Quantum Gravity 29, 124007 (2012).

[4] H. J. Kimble, Y. Levin, A. B. Matsko, K. S. Thorne, and S. P. Vyatchanin, Phys. Rev. D 65, 022002 (2001).

[5] M. Evans, L. Barsotti, P. Kwee, J. Harms, and H. Miao, Phys. Rev. D 88, 022002 (2013).

[6] T. Isogai, J. Miller, P. Kwee, L. Barsotti, and M. Evans, Opt. Express 21, 30114 (2013).

[7] E. E. Mikhailov, K. Goda, T. Corbitt, and N. Mavalvala, Phys. Rev. A 73, 053810 (2006).

[8] S. Weis, R. Riviére, S. Delèglise, E. Gavartin, O. Arcizet, A. Schliesser, and T. J. Kippenberg, Science 330, 1520 (2010).

[9] J. D. Teufel, D. Li, M. S. Allman, K. Cicak, A. J. Sirois, J. D. Whittaker, and R.W. Simmonds, Nature (London) 471, 204 (2011).
[10] T. Corbitt, Y. Chen, E. Innerhofer, H. Muller-Ebhardt, D. Ottaway, H. Rehbein, D. Sigg, S. Whitcomb, C. Wipf, and N. Mavalvala, Phys. Rev. Lett. 98, 150802 (2007).

[11] T. Corbitt, C. Wipf, T. Bodiya, D. Ottaway, D. Sigg, N. Smith, S. Whitcomb, and N. Mavalvala, Phys. Rev. Lett. 99, 160801 (2007).

[12] D. E. Chang, K.-K. Ni, O. Painter, and H. J. Kimble, New J. Phys. 14, 045002 (2012).

[13] K.-K. Ni, R. Norte, D. J. Wilson, J. D. Hood, D. E.Chang, O. Painter, and H. J. Kimble, Phys. Rev. Lett. 108, 214302 (2012).

[14] I. Wilson-Rae, N. Nooshi, W. Zwerger, and T. J. Kippenberg, Phys. Rev. Lett. 99, 093901 (2007).

[15] F. Marquardt, J. P. Chen, A. A. Clerk, and S. M. Girvin, Phys. Rev. Lett. 99, 093902 (2007).

[16] W. Z. Korth, H. Miao, T. Corbitt, G. D. Cole, Y. Chen, and R. X. Adhikari, Phys. Rev. A 88, 033805 (2013).

[17] J. D. Thompson, B. M. Zwickl, A. M. Jayich, F. Marquardt, S. M. Girvin, and J. G. E. Harris, Nature (London) 452, 72 (2008).

[18] A. M. Jayich, J. C. Sankey, B. M. Zwickl, C. Yang, J. D. Thompson, S. M. Girvin, A. A. Clerk, F. Marquardt, and J. G. E. Harris, New J. Phys. 10, 095008 (2008).

[19] See Supplemental Material at http://link.aps.org/ supplemental/10.1103/PhysRevLett.113.151102. which includes Refs. [20-22], for a detailed analysis on the optomechanical dynamics of our optical dilution scheme and a discussion on the possible thermal effect created by the optical heating.

[20] H. Miao, S. Danilishin, T. Corbitt, and Y. Chen, Phys. Rev. Lett. 103, 100402 (2009).

[21] L. Meirovitch, Analytical Methods in Vibrations (Prentice Hall, Englewood Cliffs, NJ, 1967).

[22] C. J. Glassbrenner and G. A. Slack, Phys. Rev. 134, A1058 (1964).

[23] G. Cagnoli, L. Gammaitoni, J. Hough, J. Kovalik, S. McIntosh, M. Punturo, and S. Rowan, Phys. Rev. Lett. 85, 2442 (2000). 